\title{
Material Learning
}

\author{
Wilfred G. van der Wiel \\ NanoElectronics Group, BRAINS Center for Brain-Inspired Nano Systems, \\ MESA+ Institute for Nanotechnology, University of Twente, \\ P.O. Box 217, 7500 AE Enschede, The Netherlands \\ E-mail: W.G.vanderWiel@utwente.nl
}

Natural and man-made information processing systems differ greatly. Evolution has resulted in living systems that utilize whatever physical properties are exploitable to enhance the fitness for survival. Nature thereby exploits the emergent properties and massive parallelism of highly interconnected networks of locally active components. Man-made computers, however, are based on circuits of functional units, following rigid design rules. In conventional (classical) computational paradigms, potentially exploitable physical processes to solve a problem, are possibly left out. Here, we manipulate physical systems using the principle of Material Learning, to take full advantage of the computational power of nanomaterial networks.

We have shown that a designless network of gold nanoparticles can be evolved into Boolean logic gates [1]. Now we demonstrate that the above principle is generic and can be demonstrated in other material systems as well, at much higher temperatures. By exploiting the nonlinearity of a nanoscale network of boron dopants in silicon (Si:B networks), we can significantly facilitate (nonlinear) classification. We map a limited number of input data to a new, high-dimensional feature space, in which the data become linearly separable. Using a convolutional neural network approach, it becomes possible to use our device for handwritten digit recognition [2].

We also show that our Si:B network can be well described by a deep neural network, which allows for applying standard machine learning techniques in finding functionality [3]. We argue that this approach can be helpful in optimizing complex (quantum) nanoelectronic devices in general.
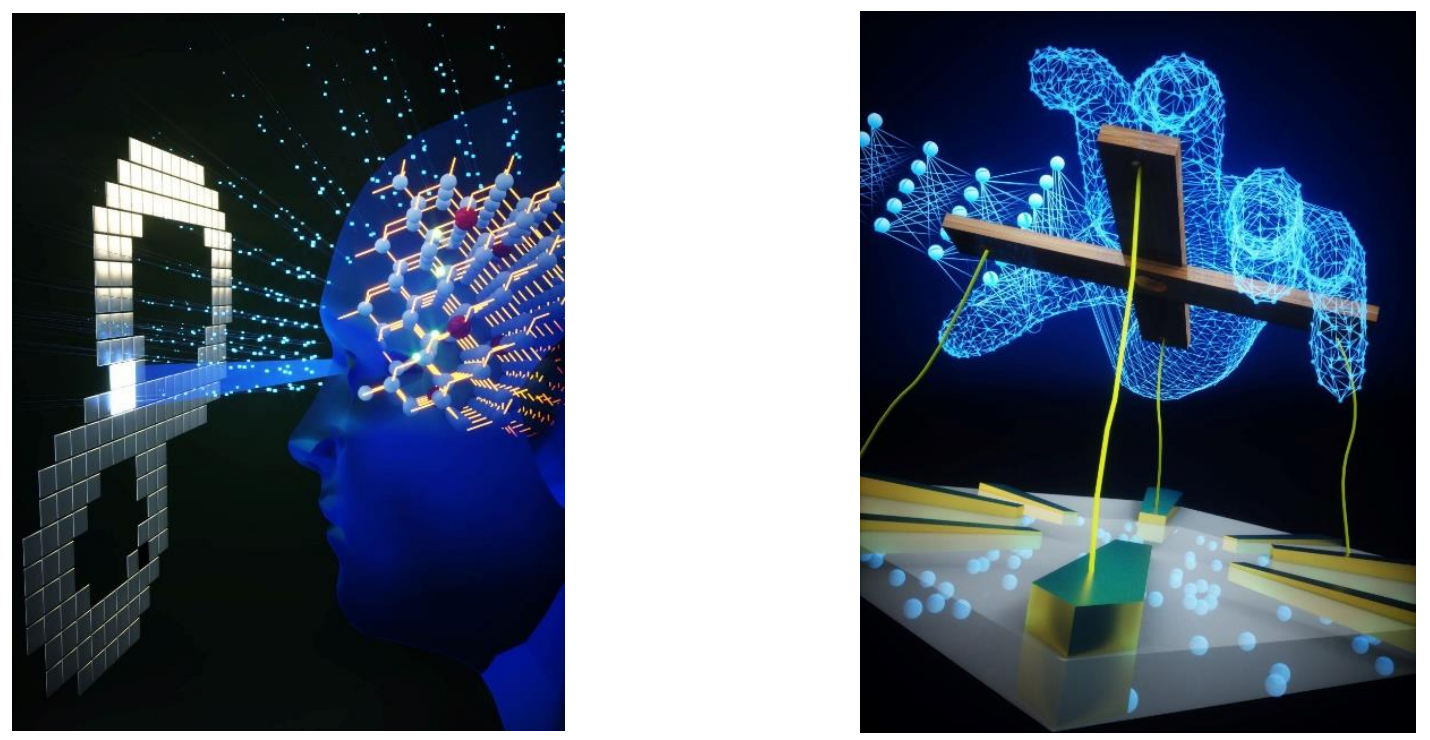


\section{References}

[1] S.K. Bose, C.P. Lawrence, Z. Liu, K.S. Makarenko, R.M.J. van Damme, H.J. Broersma and W.G. van der Wiel, Nature Nanotechnology 10, 1048 (2015).

[2] T. Chen, J. van Gelder, B. van de Ven, S. Amitonov, B. de Wilde, H.-C. Ruiz Euler, H.J. Broersma, P.A. Bobbert, F.A. Zwanenburg and W.G. van der Wiel, Nature 577, 341 (2020).

[3] H.-C. Ruiz Euler, M. N. Boon, J. T. Wildeboer, B. van de Ven, T. Chen, H.J. Broersma, P. A. Bobbert, W. G. van der Wiel, Nature Nanotechnology (2020). 